\title{
Apple Watch Steps
}

\author{
Praveen Veerabhadrappa, M.B.B.S., M.S., Ph.D. ', Matthew B. Rhudy, Ph.D. ', \\ Matthew Duffy Moran, B.S. ${ }^{1}$, Mitchell D. Renninger, B.S. ${ }^{2}$, Scott B. Dreisbach, B.S. ${ }^{7}$, and Kristin M. Gift, \\ B.S ${ }^{\top}$
}

${ }^{1}$ Kinesiology, Division of Science, The Pennsylvania State University, Reading, PA, USA; ${ }^{2}$ Engineering, Division of Engineering, Business and Computing, The Pennsylvania State University, Reading, PA, USA.

J Gen Intern Med 34(1):14

DOI: $10.1007 / \mathrm{s} 11606-018-4729-7$

(C) Society of General Internal Medicine 2018

$\mathrm{R}$ oughly, 8-10\% of US adults own an Apple Watch or some sort of wearable device. These technologies are receiving much attention for their role in tracking health behaviors and their adoption is expected to grow (https:// expandedramblings.com/index.php/apple-watch-statistics/). ${ }^{1}$

In our article, ${ }^{2}$ we reported a study evaluating the accuracy of step counts obtained from Apple Watch at four different walking speeds using a treadmill protocol and compared with manual counts of video recordings. We found that Apple Watch tested to be an extremely accurate device for measuring daily step counts. In essence, our study rules out "false negatives," i.e., Apple Watch is extremely accurate in detecting the actual steps taken while walking.

In the letter, Dr. Holleck highlights an important limitation, Apple Watch's "false positives" or incorrectly detecting arm movements as "steps." The presented idea is focused on false positives, that is, when steps are counted by other activities which may not have actually been a physical step. This might be a separate issue of activity classification which starts to complicate the issue from not just counting steps, but counting other activities as well. As in, instead of a single measure of activity (steps), there may be " $\mathrm{x}$ " number of minutes walking, " $\mathrm{x}$ " minutes running, " $\mathrm{x}$ " minutes cycling, " $\mathrm{x}$ " minutes playing piano, etc. Future activity trackers could focus on this idea of classifying various activities, since a step is not necessarily "a step." Part of the step counting measure though is as an equivalence of activity, as in " $x$ steps" on an activity tracker is supposed to be equivalent to the amount of activity of taking

Published online November 6, 2018 "x steps." This may have been achieved through means that are not just walking but any physical activity or equivalent body movement. Dr. Holleck, in the letter, is capturing some measure of the physical activity which was done while playing piano. While this amount may not be technically correct, it does indicate that the act of playing piano is measuring as physical activity.

We agree with such important limitations. Nonetheless, our study reveals three important insights. The Apple Watch proved to be extremely accurate for recording daily step counts. And, it seemed to be the most accurate at the moderate pace. This might be a practical application since the American College of Sports Medicine recommends moderate intensity exercise. Finally, our results may help reinforce individuals' trust in these devices.

Corresponding Author: Praveen Veerabhadrappa, M.B.B.S., M.S., Ph.D.; Kinesiology, Division of Science The Pennsylvania State University, 114D Beaver, Penn State Berks, Tulpehocken Road, P. O. Box 7009, Reading, PA 19610-6009, USA (e-mail: pmv5057@psu. edu).

\section{Compliance with Ethical Standards:}

Conflict of Interest: The authors declare that they do not have a conflict of interest.

\section{REFERENCES}

1. US Census Bureau. Computer and Internet use. http://www.census.gov/ hhes/computer/.

2. Veerabhadrappa, P., Moran, M. D., Renninger, M. D., Rhudy, M. B., Dreisbach, S. B., Dreisbach, S. B. \& Gift, K. M. (2018). Tracking Steps on Apple Watch at Different Walking Speeds. Journal of general internal medicine, 33(6), 795-796. 